\title{
A Trivariate Weibull Model for Oestradiol Plus Progestrone Treatment Increases Serum Leptin Concentrations in Normal Premenopasual Women
}

\author{
S. Lakshmi *And K. Sheeja** \\ *Principal (Rtd.), Kunthavai Naacchiyaar Govt. Arts College for Women,(Autonomous),Thanjavur-613007 \\ **Assistant Professor of Mathematics, SIES (Nerul) College of Arts Science and Commerce, Nerul, Navi \\ Mumbai-410210, Maharastra.
}

\begin{abstract}
In the previous studies a hypothesis was developed from the results by using trivariate Weibull model that the increase in leptin concentrations during the second half of the menstrual cycle may be related to changes in the steroidal milieu during the pre ovulatory period and the luteal phase. The present study was undertaken to test this hypothesis further by examining the effect of treatment with oestradiol and progesterone on leptine concentrations in normal pre menopausal women. The trivariate Weibull model is used for finding survival functions and log-likelihood functions for corresponding values of oestradiol, LH and Leptin for both untreated and treated with oestradiol and oestradiol plus progesterone respectively.
\end{abstract}

Keyword: Trivariate Weibull, LH, Leptin, oestradiol and progesterone

\subsection{Trivariate Weibull Distribution:}

\section{Mathematical Model}

$\mathrm{Lu}$ and Bhattacharya [5] developed a joint survival function by letting $\mathrm{h}_{1}(\mathrm{x})$ and $\mathrm{h}_{2}(\mathrm{y})$ be two arbitrary failure rate functions on $[0, \infty)$, and $\mathrm{H}_{1}(\mathrm{x})$ and $\mathrm{H}_{2}(\mathrm{y})$ be their corresponding cumulative failure rate. Given the stress $\mathrm{S}=\mathrm{s}>0$, the joint survival function conditioned on $\mathrm{s}$, as they defined is,

$\bar{F}(\mathrm{x}, \mathrm{y} / \mathrm{s})=\exp \left\{-[H 1(x)+H 2(y)]^{\gamma_{s}}\right\}$, where $\gamma$ measures the conditional association of $\mathrm{X}$ and $\mathrm{Y}$. Further, based on the joint survival function, they proved a theorem that a bivariate survival function $\bar{F}(\mathrm{x}, \mathrm{y} / \mathrm{s})$ can be derived with the marginals $\bar{F}_{\mathrm{x}}$ and $\bar{F}_{\mathrm{y}}$ given the assumption that the Laplace transform of the stress $\mathrm{S}$ exists on $[0, \infty)$ and is strictly decreasing.

From the theorem, they derived a bivariate Weibull Distribution

$$
\bar{F}(\mathrm{x}, \mathrm{y})=\exp \left\{-\left[\left(\frac{x}{\lambda_{1}}\right)^{\frac{\gamma_{1}}{\alpha}}+\left(\frac{y}{\lambda_{2}}\right)^{\frac{\gamma_{2}}{\alpha}}\right]\right\} \text { where } 0<\alpha \leq 1,0<\lambda_{1}, \lambda_{2} \leq \infty, 0<\gamma_{1}, \gamma_{2}<\infty
$$

This bivariate Weibull Distribution is exactly the same as developed by Hougaard [8].

Following the same steps the theorem can be expanded to more than random variables and, therefore, a multivariate survival function of Weibull Distribution is constructed as

$$
\mathrm{S}\left(\mathrm{x}_{1}, \mathrm{x}_{2}, \ldots \mathrm{x}_{\mathrm{n}}\right)=\exp \left\{-\left[\left(\frac{x_{1}}{\lambda_{1}}\right)^{\frac{\gamma_{1}}{\alpha}}+\left(\frac{x_{2}}{\lambda_{2}}\right)^{\frac{\gamma_{2}}{\alpha}}+\cdots\left(\frac{x_{n}}{\lambda_{n}}\right)^{\frac{\gamma_{n}}{\alpha}}\right]^{\alpha}\right\}
$$

Where $\alpha$ measures the association among the variables, $0 \leq \alpha<1$, and $0<\lambda_{1}, \lambda_{2}, \ldots . \lambda_{n}<\infty$ and $0<$ $\gamma_{1}, \gamma_{2}, \ldots . . \gamma_{n}<\infty[2,3]$

1.2 Probability Density Function of the Multivariate Weibull Distribution:

The multivariate probability density function $f\left(\mathrm{x}_{1}, \mathrm{x}_{2}, \ldots \mathrm{x}_{\mathrm{n}}\right)$ of a multivariate distribution can be obtained by differentiating the multivariate survival function with respect to each variable. Li [4], and Yi and Weng [13] had shown that

$f\left(\mathrm{x}_{1}, \mathrm{x}_{2}, \ldots \mathrm{x}_{\mathrm{n}}\right)=(-1)^{\mathrm{n}} \frac{\mathrm{n}^{\mathrm{n}} S\left(x_{1}, x_{2} \ldots x_{n}\right)}{\partial x_{1} \partial x_{2} \ldots \ldots . x_{n}}$

using Li's derivation and one of the special cases of the multivariate Faa di druno formula by Constantine and Savits [2], the probability density function is

$$
\begin{aligned}
& f\left(\mathrm{x}_{1}, \mathrm{x}_{2}, \ldots \mathrm{x}_{\mathrm{n}}\right)=\left(\frac{-1}{\alpha}\right)^{\mathrm{n}} \exp \left\{-\left[\left(\frac{x_{1}}{\lambda_{1}}\right)^{\frac{\gamma_{1}}{\alpha}}+\left(\frac{x_{2}}{\lambda_{2}}\right)^{\frac{\gamma_{2}}{\alpha}}+\cdots\left(\frac{x_{n}}{\lambda_{n}}\right)^{\frac{\gamma_{n}}{\alpha}}\right]\right\} \\
& \cdot {\left[\left(\frac{\gamma_{1}}{\lambda_{1}}\right)\left(\frac{\gamma_{2}}{\lambda_{2}}\right) \ldots\left(\frac{\gamma_{n}}{\lambda_{n}}\right)\right]\left[\left(\frac{x_{1}}{\lambda_{1}}\right)^{\frac{\gamma_{1}}{\alpha}-1}+\left(\frac{x_{2}}{\lambda_{2}}\right)^{\frac{\gamma_{2}}{\alpha}-1}+\cdots\left(\frac{x_{n}}{\lambda_{n}}\right)^{\frac{\gamma_{n}}{\alpha}-1}\right] } \\
& \sum_{i=1}^{\mathrm{p}(n)}\left\{(-1)^{k_{i}} P_{S}(n, i)\left(\prod_{i=1}^{n} \alpha^{n_{j}}-\left[\left(\frac{x_{1}}{\lambda_{1}}\right)^{\frac{\gamma_{1}}{\alpha}-1}+\left(\frac{x_{2}}{\lambda_{2}}\right)^{\frac{\gamma_{2}}{\alpha}-1}+\cdots\left(\frac{x_{n}}{\lambda_{n}}\right)^{\frac{\gamma_{n}}{\alpha}-1}\right]^{k_{i} \alpha-n}\right)\right\}
\end{aligned}
$$


where $k_{i}$ is the number of summands of the $\mathrm{i}^{\text {th }}$ partition of $\mathrm{n}$ such that $n_{1}+n_{2}+\ldots . n_{k_{i}}=\mathrm{n}, \mathrm{n}_{1} \geq \mathrm{n}_{2} \geq \cdots n_{k_{i}}>0$, $1 \leq k_{i} \leq n ; \alpha^{n_{j}}$ is equal to $(\alpha-1) \ldots .\left(\alpha-n_{j}-1\right)$, the falling of $\alpha ; \mathrm{p}(\mathrm{n})$ is the total number of set partitions of the set $S_{n}=\{1, \ldots, n\}$ corresponding to the $i^{\text {th }}$ partition of $n$.

The PDF of trivariate Weibull is

$$
\begin{aligned}
& f\left(\mathrm{x}_{1}, \mathrm{x}_{2}, \ldots \mathrm{x}_{\mathrm{n}}\right)=(-1)^{3} \frac{\partial^{3} s\left(x_{1}, x_{2}, x_{3}\right)}{\partial x_{1} \partial x_{2} \partial x_{3}} \\
& =\frac{\gamma_{1} \gamma_{1} \gamma_{1}}{\alpha^{2} x_{1} x_{2} x_{3}} \exp \left\{-\left[\left(\frac{x_{1}}{\lambda_{1}}\right)^{\frac{\gamma_{1}}{\alpha}}+\left(\frac{x_{2}}{\lambda_{2}}\right)^{\frac{\gamma_{2}}{\alpha}}+\left(\frac{x_{3}}{\lambda_{3}}\right)^{\frac{\gamma_{3}}{\alpha}}\right]^{\alpha}\right\} \\
& \cdot\left(\frac{x_{1}}{\lambda_{1}}\right)^{\frac{\gamma_{1}}{\alpha}}\left(\frac{x_{2}}{\lambda_{2}}\right)^{\frac{\gamma_{2}}{\alpha}}\left(\frac{x_{1}}{\lambda_{3}}\right)^{\frac{\gamma_{3}}{\alpha}} \cdot\left(\left(\frac{x_{1}}{\lambda_{1}}\right)^{\frac{\gamma_{1}}{\alpha}}+\left(\frac{x_{2}}{\lambda_{2}}\right)^{\frac{\gamma_{2}}{\alpha}}+\left(\frac{x_{3}}{\lambda_{3}}\right)^{\frac{\gamma_{3}}{\alpha}}\right)^{-3+\alpha} \\
& \cdot\left(2+3 \alpha\left(-1+\left(\left(\frac{x_{1}}{\lambda_{1}}\right)^{\frac{\gamma_{1}}{\alpha}}+\left(\frac{x_{2}}{\lambda_{2}}\right)^{\frac{\gamma_{2}}{\alpha}}+\left(\frac{x_{3}}{\lambda_{3}}\right)^{\frac{\gamma_{3}}{\alpha}}\right)\right)^{\alpha}+\alpha^{2}\left(1-3\left[\left(\frac{x_{1}}{\lambda_{1}}\right)^{\frac{\gamma_{1}}{\alpha}}+\left(\frac{x_{2}}{\lambda_{2}}\right)^{\frac{\gamma_{2}}{\alpha}}+\left(\frac{x_{3}}{\lambda_{3}}\right)^{\frac{\gamma_{3}}{\alpha}}\right]^{\alpha}+\right.\right. \\
& \left.\left.\left[\left(\frac{x_{1}}{\lambda_{1}}\right)^{\frac{\gamma_{1}}{\alpha}}+\left(\frac{x_{2}}{\lambda_{2}}\right)^{\frac{\gamma_{2}}{\alpha}}+\left(\frac{x_{3}}{\lambda_{3}}\right)^{\frac{\gamma_{3}}{\alpha}}\right]^{2 \alpha}\right)\right) \\
& \sum_{i=1}^{n} \log \left(f\left(x_{1_{i}}, x_{2_{i}}, x_{3_{i}}\right)\right)= \\
& \sum_{i=1}^{n} \log \left(\frac{\gamma_{1} \gamma_{1} \gamma_{1}}{\alpha^{2} x_{1} x_{2_{i}} x_{3_{i}}}\right)- \\
& \sum_{i=1}^{n}\left(\left(\frac{x_{1}}{\lambda_{1}}\right)^{\frac{\gamma_{1}}{\alpha}}+\left(\frac{x_{2_{i}}}{\lambda_{2}}\right)^{\frac{\gamma_{2}}{\alpha}}+\left(\frac{x_{3_{i}}}{\lambda_{3}}\right)^{\frac{\gamma_{3}}{\alpha}}\right)^{\alpha}+\sum_{i=1}^{n} \log \left(\left(\frac{x_{1_{i}}}{\lambda_{1}}\right)^{\frac{\gamma_{1}}{\alpha}}\left(\frac{x_{2_{i}}}{\lambda_{2}}\right)^{\frac{\gamma_{2}}{\alpha}}\left(\frac{x_{3_{i}}}{\lambda_{3}}\right)^{\frac{\gamma_{3}}{\alpha}}\left(\left(\frac{x_{1_{i}}}{\lambda_{1}}\right)^{\frac{\gamma_{1}}{\alpha}}\left(\frac{x_{2_{i}}}{\lambda_{2}}\right)^{\frac{\gamma_{2}}{\alpha}}\left(\frac{x_{3_{i}}}{\lambda_{3}}\right)^{\frac{\gamma_{3}}{\alpha}}\right)^{\alpha}\right)+ \\
& \sum_{i=1}^{n} \log \left(2+3 \alpha\left(-1+\left(\left(\frac{x_{1_{i}}}{\lambda_{1}}\right)^{\frac{\gamma_{1}}{\alpha}}\left(\frac{x_{2_{i}}}{\lambda_{2}}\right)^{\frac{\gamma_{2}}{\alpha}}\left(\frac{x_{3_{i}}}{\lambda_{3}}\right)^{\frac{\gamma_{3}}{\alpha}}\right)^{\alpha}\right)+\right. \\
& \left.\alpha^{2}\left(1-3\left(\left(\frac{x_{1_{i}}}{\lambda_{1}}\right)^{\frac{\gamma_{1}}{\alpha}}\left(\frac{x_{2_{i}}}{\lambda_{2}}\right)^{\frac{\gamma_{2}}{\alpha}}\left(\frac{x_{3_{i}}}{\lambda_{3}}\right)^{\frac{\gamma_{3}}{\alpha}}\right)^{\alpha}+\left(\left(\frac{x_{1_{i}}}{\lambda_{1}}\right)^{\frac{\gamma_{1}}{\alpha}}\left(\frac{x_{2_{i}}}{\lambda_{2}}\right)^{\frac{\gamma_{2}}{\alpha}}\left(\frac{x_{3_{i}}}{\lambda_{3}}\right)^{\frac{\gamma_{3}}{\alpha}}\right)^{2 \alpha}\right)\right)
\end{aligned}
$$

\section{Introduction}

Normal women have significantly higher serum leptin concentrations than normal men [12]. Also in the luteal phase of the normal menstrual cycle, serum leptin concentration are significantly higher than in the follicular phase [7,9], while significant positive correlations have been found between leptin and oestrodiol or progesterone concentrations[10]. Although these studies suggest that ovarian steroids may play a role in leptin secretion in humans no direct evidence has been provided as yet. A significant decrease in serum leptin concentration was found following bilateral ovariectomy in normal women [10], and although treatment with oestradiol was without any effect, the addition of progesterone prevented this decrease, suggesting that progesterone plays an important role in the control of leptin secretion [11]. A hypothesis was developed from these results that the increase in leptin concentration during the second half of the menstrual cycle may be related to changes in the steroidal milieu during the periovulatory period and the luteal phase. The present study was undertaken to test this hypothesis further by examining the effect of treatment with oestrodiol and progesterone on leptin concentration in normal premenopausal women.

\section{Results And Discussion}

Serum concentrations of oestradiol, progesterone, Leptin, and LH during the administration of oestradiol (oestradiol cycles)and the control cycles in the 10 women are shown in Figure [1,2,3]. Concentrations of these hormones on cycle day 2 were similar between the oestradiol and the control cycles. A marked increase in oestradiol concentrations was seen in all women from the onset of treatment on cycle day 2 to day 5. Subsequently, oestradiol concentrations declined rapidly and returned to pretreatment level on cycle day 7, and showed no significant changes thereafter. In the control cycle, serum oestradiol concentrations increased slightly from days 2 to 7 and progressively from days 7 to 10. From days 3 to 6 oestradiol concentrations was slightly higher, and on days 9 and 10 were slightly lower, in the oestradiol cycle than in control cycle. Serum progesterone concentrations were low throughout the experimental period in both the oestradiol and control cycle. The changes in serum oestradiol, progesterone Leptin, and LH concentrations in the six cycles treated with oestradiol plus progesterone and in the corresponding control cycles are shown in Figure $[4,5,6]$. Pretreatment concentrations of these hormones on cycle day 2 did not differ significantly between the two groups of cycle. During treatment with oestradiol and progesterone serum oestradiol concentration showed a pattern of increase similar to that in oestradiol cycle, with no significant difference at 
the corresponding points. Oestradiol concentrations were significantly higher on days 3, 4, and 5 and significantly lower on days 9 and 10 in the oestradiol plus progesterone cycles than in the control cycles. Serum progesterone concentrations in the oestradiol + progesterone cycles showed an abrupt increase from days 3 to 4 , remaining high on days 5 and 6 and decreasing thereafter.

This study is first to show an increase in serum leptin concentrations in normal women during treatment with exogenous oestradiol and progesterone. It is of interest that when oestradiol alone was given to the women there was no change in leptin concentration, but the increase was observed when progesterone was added to the oestradiol regimen. This suggests that in the presence of progesterone and normal oestradiol the leptin secretion increases, but however whether the progesterone has the same effect in the presence of low oestradiol needs to be investigated. The aim of this study was to examine whether a sequence of events in terms of changes in oestradiol and progesterone concentrations resembling those in the normal menstrual cycle, can affect leptin secretion. The increase in leptin concentration during treatment with oestrodiol + progesterone was seen in all women. During treatment with oestradiol and progesterone, serum concentration of oestradiol showed a pattern of increase similar to that seen during the preovulatory period of the normal menstrual cycle, and although progesterone concentration showed an abrupt increase, the concentration was similar to those seen in luteal phase of the cycle. The importance of increased serum leptin concentration during the luteal phase of the cycle is unclear. It has been speculated that leptin at that stage may play a role in embryo implantation, possibly through a mechanism that affects the invasion phase. Alternatively the increase in leptin concentrations may help the body to meet the metabolic demand of pregnancy. In terms of possible impact that progestrone induced increase in leptin concentration might have in clinical practice.

\section{Medical Figures}

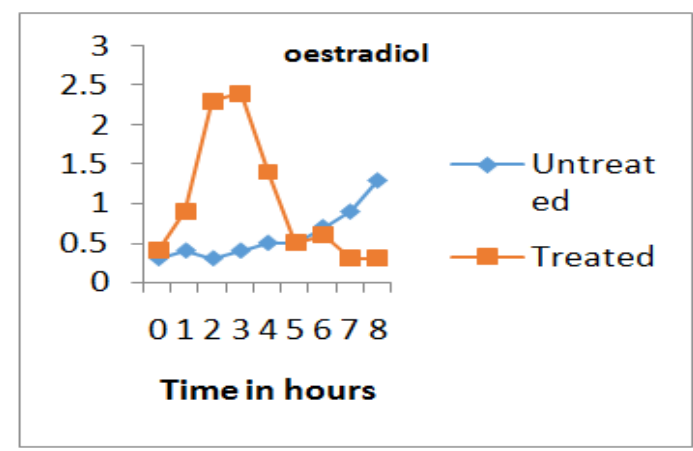

Figure 1

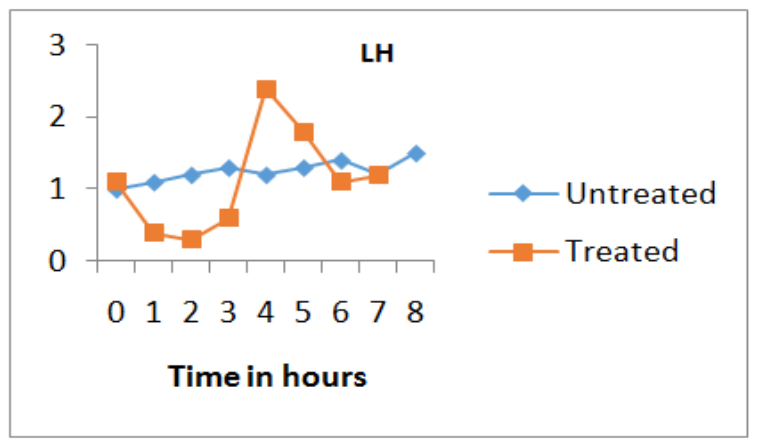

Figure 2

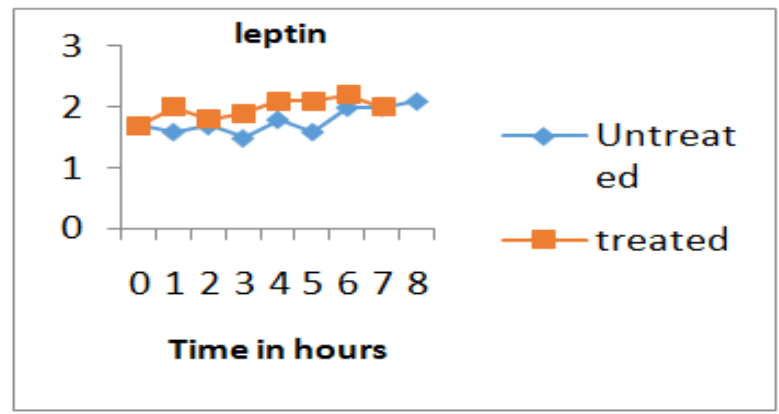

Figure 3 


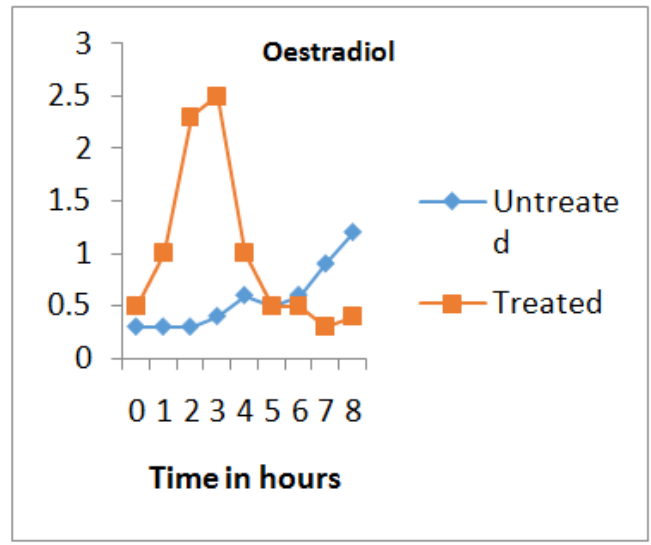

Figure 4

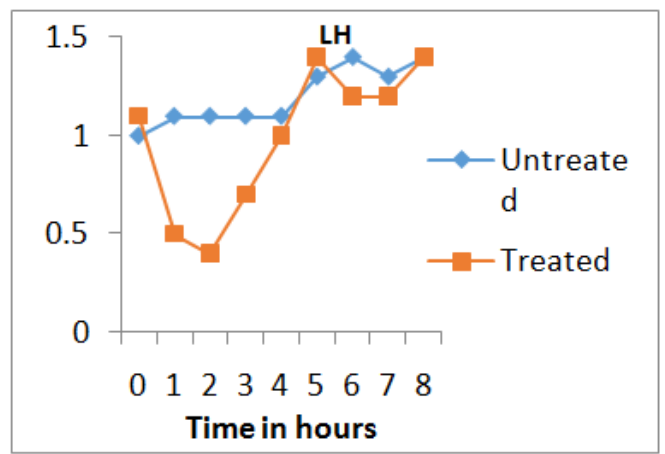

Figure 5

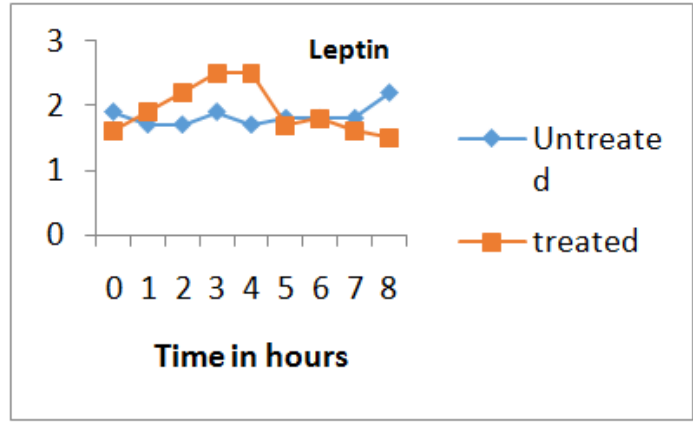

Figure 6

Figure[1,2,3]: Serum oestradiol, progesterone,LH and Leptin concentration during the follicular phase untreated spontaneous cycles and cycles treated with oestradiol through skin patches in normally cycling women.

Figure[4,5,6]: Serum oestradiol, progesterone,LH and Leptin concentration during the follicular phase untreated spontaneous cycles and cycles treated with oestradiol through skin patches plus progesterone intravaginally in normally ovulating women.

\section{Conclusion}

The study shows that preovulatory concentration of oestrodiol induced in early follicular phase of the cycle by the administration of estradiol. However induction of luteal phase concentration of progesterone in addition to the increased concentration of oestrodiol can stimulate leptin secretion. These findings may provide an explanation for the previously reported increased leptin concentrations that occur during the second half of the normal menstrual cycle. Mathematical Figure 9 and 10 shows the survival function of combined effect of LH, Leptin and Oestradiol with administration of oestradiol and oestrodiol plus progesterone for the untreated and treated cases. Figure 7 and 8 show that the Log-likelihood function of combined effect of LH, leptin and Oestradiol with administration of oestradiol and oestrodiol plus progesterone for the untreated and treated cases. By both the methods the concentration of leptin increases when treated with oestrodiol and progesterone. 
Mathematical Figures:

Log-likelihood function graph

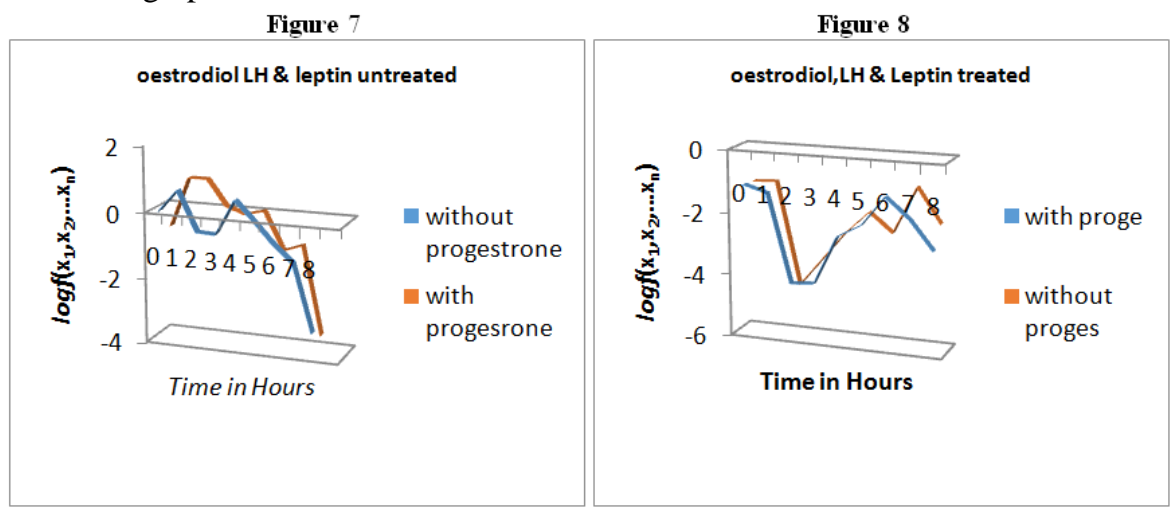

Figure [7,8]: Serum oestradiol, progesterone,LH and Leptin concentration during the follicular phase untreated spontaneous cycles and cycles treated with oestradiol through skin patches in normally cycling women.

\section{Survival function graphs}

Figure 9

Figure 10

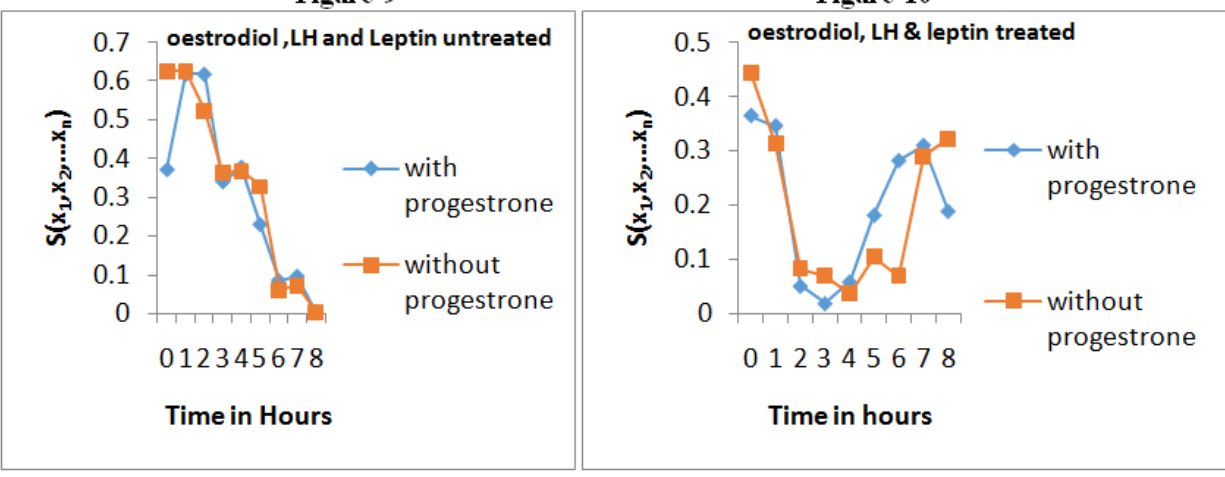

Figure[9,10]: Serum oestradiol, progesterone,LH and Leptin concentration during the follicular phase untreated spontaneous cycles and cycles treated with oestradiol through skin patches plus progesterone intravaginally in normally ovulating women.

\section{References}

[1]. Constantine, G.M., Savits, T.H., (1996). A multivariate Faa Di Bruno Formula with Applications. American Mathematical Society, Vol 358, pp 503-520.

[2]. Crowder, M., (1989). A Multivariate Distribuition with Weibull Connections. Journal of the Royal Statistical Society, Series B, Vol 51, pp 3-107.

[3]. Frees, E. W., Valdez, E. A., (1998).Understanding Relationships using Copulas. North American Actuarial Journal Vol 2, pp 1-25.

[4]. Li, C.L., (1997). A Model for Informative Censoring. Ph.D. Dissertation: The University Alabama at Birmingham.

[5]. L u, J-C., Bhattacharya, G., (1990). Some New Constructionsof Bivariate Weibull Models. Annals of the Institute of Statistical Mathematics, Vol 42, pp 543-559.

[6]. Ludwig. M., Klein, H.H., Diedrich, K. and Ortmann, O. (2000). Serum leptin concentrations throughout the menstrual cycle. Arch. Gynecol. Obstet., pp 263. -101.

[7]. Hardie, L.,Trayhum, P., Abramovich, D. and Fowler, P. (1997) Circulating leptin in women: a longitudinal study in the menstrual cucle and during pregency. Clin. Endocrinol., Vol 47, pp 101-106

[8]. Hougaard, P., (1986) A Class of Multiariate Failure Time Distributions Biometrica Vol 73, pp 671-678.

[9]. Mannucci, E., Ognibene, A., Becorpi, A. et al. (1998) Relationship between leptin and oestrogen in healthy women. Eur. J. Endocrinol., Vol 139, pp 18-201

[10]. Messinis, I.E., Milingos, S.D., Alexandris, E. et al. (1999) Leptin concentration in normal women following bilateral ovariectomy. Hum. Repro., Vol 14, pp 913-918

[11]. Messinis, I.E., Kariotis, I., Milingos, S, et al. (2000) Treatment of normal women with oestrodiol plus progesterone prevents the decrease of leptin concentrations induced by ovariectomy. Hum. Reprod., Vol 15, pp 2383-2387

[12]. Rosenbaum, M., Nilcoson, M., Hirsch, J et al. (1996) Effects of gender, body composition and menopause on plasma concentration of leptin. J. Clin. Endocrinol.Metab., Vol 81, pp 3424-3427.

[13]. Yi, Z.,Weng, C., (2006). An Application of the Power Approximation in Multiple Life Insurance, Mathematics and Economics, Vol 38 , pp 98-112. 